\title{
Extracting information from partially depleted Si detectors with digital sampling electronics
}

G. Pastore ${ }^{1,2}$, G. Pasquali ${ }^{1,2}$, N. Le Neindre ${ }^{3}$, G. Ademard ${ }^{4}$, S. BARlini ${ }^{1,2}$, M. Bini ${ }^{1,2}$, E. Bonnet ${ }^{5}$, B. Borderie ${ }^{4}$,

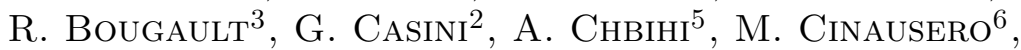
J.A. Dueñas ${ }^{7}$, P. Edelbruck ${ }^{4}$, J.D. Frankland ${ }^{5}$, F. Gramegna $^{6}$,

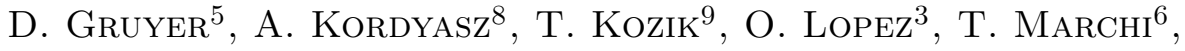
L. Morelli ${ }^{10}$, A. Olmi ${ }^{2}$, A. Ordine ${ }^{11}$, M. PÂrlog ${ }^{3,12}$, S. Piantelli ${ }^{2}$, G. Pogai ${ }^{1,2}$, M.-F. Rivet ${ }^{4}$, E. Rosato ${ }^{11,13}$, F. Salomon ${ }^{4}$, G. Spadaccini ${ }^{11,13}$, A.A. Stefanini ${ }^{1,2}$, S. VAldré ${ }^{1,2}$, E. Vient $^{3}$, T. Twaróg ${ }^{9}$, R. Alba ${ }^{14}$, C. Maiolino ${ }^{14}$ and D. Santonocito ${ }^{14}$

${ }^{1}$ Dipartimento di Fisica, Università di Firenze, Firenze, Italy

2 INFN, Sezione di Firenze, Firenze, Italy

${ }^{3}$ LPC, IN2P3-CNRS, ENSICAEN and Université de Caen, France

${ }^{4}$ IPN, CNRS/IN2P3, Université Paris Sud 11, Paris, France

${ }^{5}$ GANIL, CEA/DSM-CNRS/IN2P3, Caen, France ${ }^{6}$ INFN-LNL Legnaro (PD), Italy

${ }^{7}$ Dep. de Fisica Aplicada, FCCEE Univ. de Huelva, Huelva, Spain

${ }^{8}$ Heavy Ion Laboratory, University of Warsaw, Warsaw, Poland ${ }^{9}$ Jagiellonian Univ., Institute of Nuclear Physics IFJ-PAN, Kraków, Poland ${ }^{10}$ INFN, Sezione di Bologna and Università di Bologna, Italy

${ }^{11}$ INFN, Sezione di Napoli, Napoli, Italy 12 NIPNE-HH, Măgurele-Bucharest, Romania

${ }^{13}$ Dipartimento di Fisica, Università di Napoli "Federico II", Napoli, Italy ${ }^{14}$ INFN-LNS, Catania, Italy 


\begin{abstract}
A study of the identification properties and of the energy response of a Si-Si-CsI(Tl) $\Delta E$ - $E$ telescope exploiting a partially depleted second Si stage has been performed. Five different bias voltages have been applied to the second stage of the telescope, one corresponding to full depletion, the others associated with a depleted layer ranging from $60 \%$ to $90 \%$ of the detector thickness. Fragment identification has been obtained using either the $\Delta E-E$ technique or the Pulse Shape Analysis (PSA). Charge collection efficiency has been evaluated. The $\Delta E-E$ performance is not affected by incomplete depletion. Isotopic separation capability improves at lower bias voltages with respect to full depletion, though charge identification thresholds increase.
\end{abstract}

In recent years, intensive experimental work of the FAZIA Collaboration $[1,2]$ has been devoted to improving the nuclear fragment identification based on Pulse Shape Analysis. Since an improvement of PSA isotopic identification capabilities in partially depleted Si detectors had been observed in preliminary tests, a systematic study of PS identification capabilities of underdepleted Si detectors has been undertaken [3].

The second stage of a standard FAZIA telescope, Si2, has been biased at five different voltages (see tab.1). In fact, the PSA performance can be better studied on Si2 since charge and mass of the fragments stopped in Si2 can be obtained from the $\Delta E-E$ technique applied to fragments that punch through the first stage of the telescope.

Table 1: Main features of the detector at different bias voltages.

\begin{tabular}{|l|ccccc|}
\hline Voltage (V) & 105 & 130 & 200 & 235 & 290 \\
\hline Depletion depth $(\mu \mathrm{m})$ & 310 & 340 & 420 & 460 & 510 \\
\hline Undepleted layer $(\mu \mathrm{m})$ & 200 & 170 & 90 & 50 & 0 \\
\hline
\end{tabular}

The data were collected in Catania at Laboratori Nazionali del Sud (LNS) of INFN. The beam was ${ }^{84} \mathrm{Kr}$ at $35 \mathrm{~A} \mathrm{MeV}$ impinging on ${ }^{112} \mathrm{Sn}$ and ${ }^{197} \mathrm{Au}$ targets. A telescope composed of Si $300 \mu \mathrm{m}$-Si $500 \mu \mathrm{m}$-CsI(Tl) $10 \mathrm{~cm}$, was mounted in the Ciclope scattering chamber, at a distance from target of $50 \mathrm{~cm}$ and at $8^{\circ}$ polar angle with respect to the beam line, covering about $2^{\circ}$ in polar angle. According to the usual FAZIA recipe for optimizing the PSA performance, both silicon detectors were mounted with the ohmic (low field) side facing the target [4]. The Si detectors were of the neutron transmutation doped type for a better doping uniformity and cut at a random direction to avoid channeling effects [5]. They where connected to PACI preamplifiers placed under vacuum very close to the detectors. Signals were 
then brought outside vacuum and connected to custom made digitizers [2]. For Si2, since charge collection times in an underdepleted detector can be longer than at full depletion, the length of acquired signals has been set to $70 \mu s$ (maximum length allowed by the FEE signal memory).

An accurate study of the charge collection efficiency showed for the two lowest biases a dependence on the range of the stopped fragments: the higher the range of the projectile, the better the collection efficiency of the detector (about $98 \%$ for particles that punch through Si2). We find that it is possible to reproduce the behaviour of the collection efficiency with a simple formula exploiting the variation of the stopping power along the particle track. Details can be found in [3].
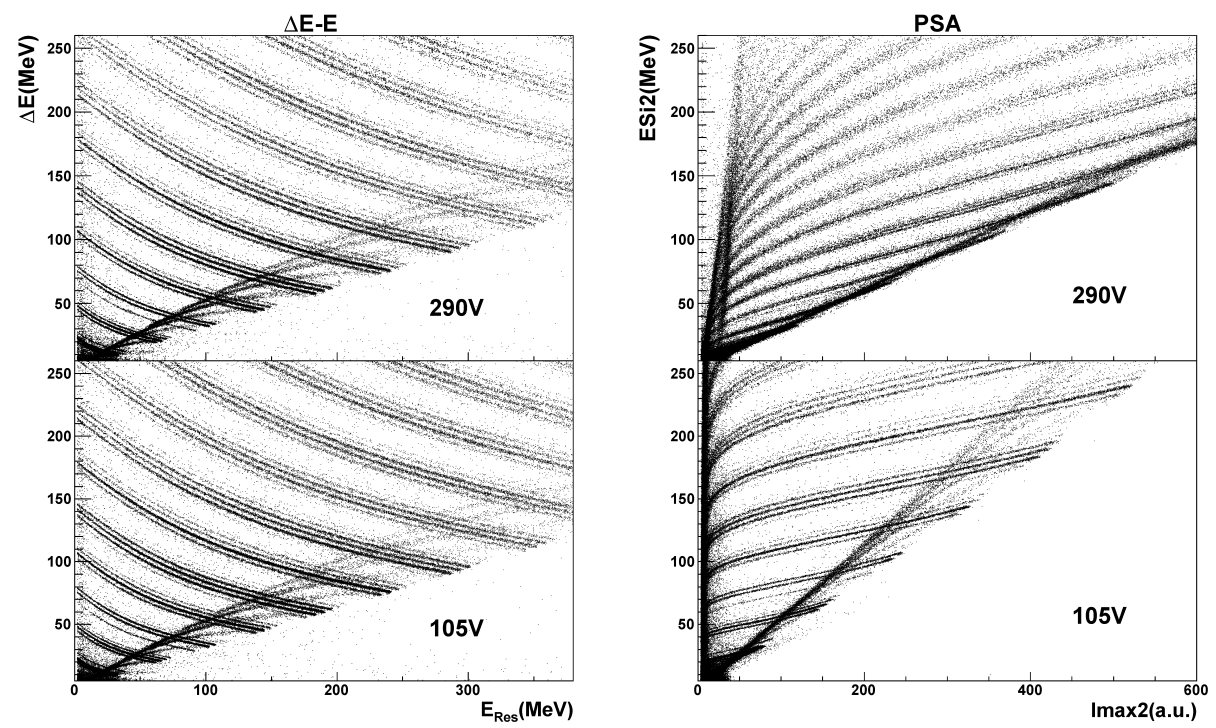

Figure 1: $\Delta E-E$ and PSA correlations obtained at the highest (top panels) and lowest (bottom panels) bias voltages.

Coming to the $\Delta E-E$ identification of fragments, the left panels of fig.1 show the correlations obtained at the highest (top panel) and lowest (bottom panel) bias voltages applied to Si2. No significant degradation of the isotopic separation can be spotted in the figure though at $105 \mathrm{~V}$, Si2 is only depleted by $60 \%$. The right panels of fig. 1 show the PSA correlations ("Energy in Si2 vs maximum of current signal") obtained at the highest (top panel) and lowest (bottom panel) bias voltages. The comparison evidences a sizable improvement of the identification capabilities of the detector when underbiased. 
The energy threshold for discrimination can be quantitatively evaluated for each element by calculating a Figure of Merit as a function of fragment energy $[2,3]$. Figure 2 presents the energy threshold for identification both in mass (left panel) and in charge (right panel) as a function of the atomic number Z. One can observe that the lower the applied voltage, the higher the energy threshold for charge identification for a given $\mathrm{Z}$ and the larger the maximum $\mathrm{Z}$ for which isotopic identification is achieved. A doping uniformity of about 1\% FWHM, or less, would be needed to discriminate isotopes via PSA in a totally depleted detector [6]. The doping uniformity of the detector under test is about $6 \%$ : in fact only when underbiased it has been found capable of identifying isotopes, even though in a reduced energy domain.
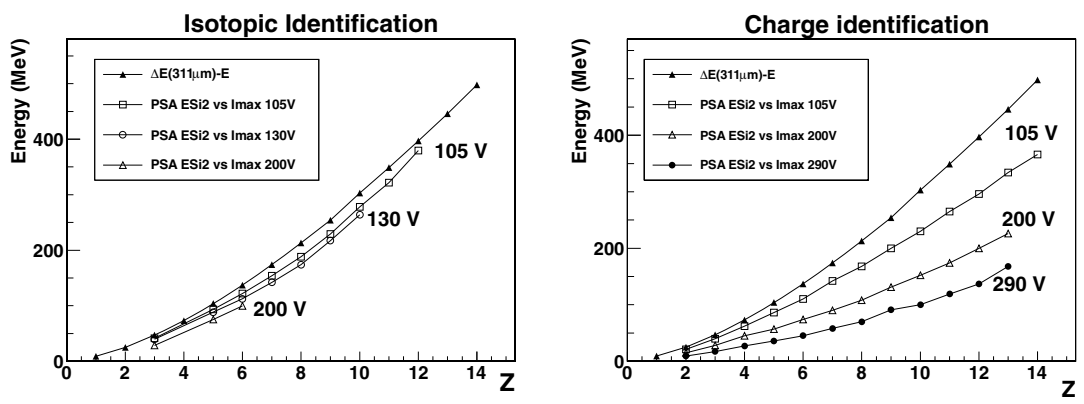

Figure 2: The energy thresholds respectively for isotopic identification on the left, for charge identification on the right.

As a conclusion, we observed a relatively high charge collection efficiency (more than about $90 \%$ ) even when only $60 \%$ of the detector thickness is depleted, identification via $\Delta E-E$ is not affected by underbias, isotopic identification via PSA improves with underbias though one gets higher energy thresholds for charge identification with respect to full depletion.

\section{References}

[1] FAZIA Collaboration, http://fazia2.in2p3.fr/spip

[2] R. Bougault et al., Eur. Phys. J. A, 50 (2014) 47.

[3] G.Pasquali et al., Eur. Phys. J. A, 50 (2014) 86.

[4] N.Le Neindre et al., Nucl. Instrum. Methods A, 701 (2013) 145.

[5] S.Carboni et al., Nucl. Instrum. Methods A, 664 (2012) 251.

[6] L.Bardelli et al., Nucl. Instrum. Methods A, 654 (2011) 272. 